\title{
Refeeding Syndrome: An Important Complication Following Obesity Surgery
}

\author{
Sonja Chiappetta ${ }^{a}$ Jürgen Stein ${ }^{b, c}$ \\ aDepartment of Obesity and Metabolic Surgery, Sana Klinikum Offenbach, Offenbach, \\ Germany; ${ }^{b}$ Clinical Nutrition, DGD Clinics Sachsenhausen, Frankfurt/M., Germany; \\ 'ICCC Rhein-Main, Frankfurt/M., Germany
}

\section{Key Words}

Refeeding syndrome · Biliopancreatic diversion · Obesity surgery

\begin{abstract}
Background: Refeeding syndrome (RFS) is an important and well-known complication in malnourished patients, but the incidence of RFS after obesity surgery is unknown and the awareness of RFS in obese patients as a postsurgical complication must be raised. We present a case of RFS subsequent to biliopancreatic diversion in a morbidly obese patient. Case Report: A 48 -year-old female patient with a BMI of $41.5 \mathrm{~kg} / \mathrm{m}^{2}$ was transferred to our hospital due to Wernicke's Encephalopathy in a global malabsorptive syndrome after biliopancreatic diversion. Parenteral nutrition, vitamin supplementation and high-dosed intravenous thiamine supplementation were initiated. After 14 days, the patient started to develop acute respiratory failure, and neurological functions were impaired. Blood values showed significant electrolyte disturbances. RFS was diagnosed and managed according to the NICE guidelines. After 14 days, phosphate levels had returned to normal range, and neurological symptoms were improved. Conclusion: Extreme weight loss following obesity surgery has been shown to be associated with undernutrition. These patients are at high risk for evolving RFS, even though they may still be obese. Awareness of RFS as a postsurgical complication, the identification of patients at risk as well as prevention and correct management should be routinely performed at every bariatric center.


Chiappettaa and Steinb,c: Refeeding Syndrome: An Important Complication Following Obesity Surgery

\section{Introduction}

Due to the worldwide epidemic of obesity, bariatric procedures are increasing in frequency and are among the most commonly performed gastrointestinal operations today. In 2011, Roux-en-Y gastric bypass (RYGB) was the most common bariatric procedure (46.6\%), followed by sleeve gastrectomy (SG; 27.8\%), adjustable gastric banding (AGB; 17.8\%), and biliopancreatic diversion/duodenal switch (BPD/DS; 2.2\%) [1]. Early and late postoperative morbidity is associated with both surgical and nutritional complications. Macro- and micronutrient deficiencies are common in obese patients even before surgery and are frequent complications following obesity surgery, dependent on the bariatric procedure used [2]. While patients with BPD as a malabsorptive procedure achieve the best results in terms of excess weight loss (EWL) - up to 70\% during a 15-year follow-up period [3] -, nutritional deficiencies are common after malabsorptive procedures [4], with protein malnutrition occurring in $7 \%$. As a result, $1.7 \%$ of these patients require surgical revision in the form of common limb elongation or restoration [3].

Refeeding syndrome (RFS) is an important complication known to occur in malnourished patients with a subnormal BMI after oral, parenteral and enteral resumption of nutrition. RFS reflects the change from catabolic to anabolic metabolism. During refeeding, glycemia leads to increased insulin and decreased secretion of glucagon. Insulin stimulates glycogen, fat, and protein synthesis, a process requiring minerals such as phosphate and magnesium, and cofactors such as thiamine. The consequence is a fall in serum levels of phosphate, potassium, and magnesium. This severe electrolyte disturbance can cause a life-threatening condition [5], and early recognition is vital in order to reduce morbidity and mortality [6]. Major risk factors for the development of RFS, according to NICE guidelines, are a BMI $<16 \mathrm{~kg} / \mathrm{m}^{2}$, unintentional weight loss of $>15 \%$ over the last 3-6 months, little or no nutritional intake for more than 10 days, and low levels of phosphate, potassium, or magnesium prior to feeding. Minor risk factors are specified as a BMI $<18.5 \mathrm{~kg} / \mathrm{m}^{2}$, unintentional weight loss of $10-15 \%$ over the last 3-6 months, little or no nutritional intake for more than 5 days, and a history of alcohol abuse or chronic use of diuretics, antacids, chemotherapy or insulin [7]. Thus, refeeding syndrome primarily occurs in patients with anorexia. A recently published multicenter study from France reported an incidence of $10 \%$ in these patients [8].

Although at first glance obese patients may not appear to be undernourished but quite the opposite, a significant risk of RFS can arise due to chronic weight loss after obesity surgery, with an EWL of approximately $60-80 \%$ not uncommon in the postoperative course. The true incidence of RFS after obesity surgery is unknown, and current literature describes only three isolated cases of RFS after obesity surgery. One late death due to malnutrition and RFS after BPD/DS was reported in 1997 [9] while Silk et al. described two further cases in 2011 [10]. The first of these case reports describes RFS in a patient with slippage after gastric banding, with long-lasting dysphagia before diagnosis and a BMI of $17.4 \mathrm{~kg} / \mathrm{m}^{2}$. The patient underwent laparoscopic removal of the gastric banding and developed RFS postoperatively after receiving $450 \mathrm{kcal}$ in the first $24 \mathrm{~h}$ and $1500 \mathrm{kcal} /$ day over the following 2 days. The second report describes a patient who underwent RYGB at a BMI of $50 \mathrm{~kg} / \mathrm{m}^{2}$ and presented with peptic ulceration of the gastrojejunal anastomosis 8 weeks after surgery. Four months after surgery, the patient had an EWL of $52 \%$ (BMI $37 \mathrm{~kg} / \mathrm{m}^{2}$ ) and a disabling fear of taking anything orally owing to pain experienced when swallowing. In accordance with NICE guidelines, enteral nutrition was reintroduced at a rate of $5 \mathrm{kcal} / \mathrm{kg}$ daily. However, despite adherence to recommendations, the patient developed RFS within $24 \mathrm{~h}$.

In this clinical case report, we describe the fourth case of RFS in a patient after BPD. In this case, RFS occurred after enteral nutrition, with typical neurological and pulmonal symptoms resolving after exact diagnosis and treatment. 
Chiappettaa and Steinb,c: Refeeding Syndrome: An Important Complication Following Obesity Surgery

\section{Case Report}

A 48-year-old female patient with a BMI of $41.5 \mathrm{~kg} / \mathrm{m}^{2}$ (height $1.68 \mathrm{~m}$, weight $117 \mathrm{~kg}$ ) was transferred to our bariatric center due to protracted diarrhea and vomiting associated with progressive apathy and weakness due to lithium overdose. The patient was known to be taking lithium-containing medication on the basis of her known depression. The diagnosis was made by colleagues from the neurological hospital from which she had been transferred. The patient's bariatric history started in 2003, when she had undergone laparoscopic gastric banding at an initial weight of $174 \mathrm{~kg}$ (BMI $61.6 \mathrm{~kg} / \mathrm{m}^{2}$ ). Gastric banding was removed in 2005 due to slippage, and in 2009 the patient presented again at the initial weight of $174 \mathrm{~kg}$. Indication for BPD was given, and BPDScopinaro was performed laparoscopically. Three years after surgery, the patient showed an EWL of 83.96\%, but was additionally suffering from manifest hypoproteinemia associated with edema and chronic dermatoses. Initial nutritional monitoring and supplementation brought no improvement, and indication for laparoscopic conversion in BPD-Larrad was given, aiming to reduce protein malabsorption. Surgery was performed in 2012 at a weight of $85 \mathrm{~kg}\left(\right.$ BMI $\left.30.1 \mathrm{~kg} / \mathrm{m}^{2}\right)$. The postoperative course was uneventful, and follow-up in 2013 showed normal values of total serum protein and a weight of $98 \mathrm{~kg}$. Due to her known depression and noncompliance the patient did not present from 2013 to 2014 for follow-up consultation, as a result of which no supplementation was performed during this year. The patient reported that her diet was scarce of protein.

On admission to our bariatric center in 2014, the patient presented with a global malabsorptive syndrome, with deficiencies of vitamin $\mathrm{B}_{1}, \mathrm{~B}_{12}, \mathrm{~B}_{6}, \mathrm{D}, \mathrm{K}$, zinc, selenium, and iron, and with severe hypoproteinemia. The patient was in a state of mental confusion and was found to have gait disorders and acute ophthalmoplegia with diplopia. MRI scan of the brain showed no pathological findings of hemorrhagic or ischemic stroke. The classic triad of ophthalmoplegia, gait and stance disorder, together with mental confusion associated with vitamin $\mathrm{B}_{1}$ deficiency, led to the diagnosis of Wernicke's encephalopathy. Parenteral nutrition, vitamin supplementation, and high dose intravenous thiamine supplementation (300 $\mathrm{mg}$ /day) were initiated.

Due to protracted vomiting and diarrhea, an upper gastrointestinal endoscopy was performed. This was negative for stenosis. A stool culture showed no abnormal bacteria or parasites in the sample. Treatment with parenteral, and then enteral, nutrition via a nasogastric tube at a rate of $1,200 \mathrm{kcal} /$ day $(10 \mathrm{kcal} / \mathrm{kg}) \mathrm{was}$ initiated according to the NICE guidelines for patients at high risk for RFS.

During the course of the next 10 days, the patient started to develop acute respiratory failure due to pulmonary edema, necessitating non-invasive ventilation. Neurological functions were impaired. Blood values showed significant electrolyte disturbances, including hypopotassemia, hypomagnesemia and severe hypophosphatemia of $0.9 \mathrm{mg} / \mathrm{dl}(0.05 \mathrm{mmol} / \mathrm{l})$ (range $2.5-4.5 \mathrm{mg} / \mathrm{dl}$ ).

RFS was managed in accordance with NICE guidelines, with appropriate fluid balance and micronutrient replacement aimed at the correction of potassium, phosphate, calcium and magnesium, with intravenous supplementation of $18 \mathrm{mmol}$ phosphate over $12 \mathrm{~h}$ [7]. Diuretic therapy was started in order to treat the pulmonary edema.

After 14 days, phosphate levels had returned to the normal range (3.8 mg/dl) and neurological symptoms had improved. The patient showed no further mental confusion and was able to begin self-mobilization. At this point, she began a progressive oral diet with nutritional supplements, which was well tolerated. All pulmonary and neurological symptoms resolved following treatment.

Indication for revisional surgery was given, and 1 month after RFS, the patient underwent open revision from BPD-Larrad to RYGB with an alimentary limb of $150 \mathrm{~cm}$, a biliopancreatic limb of $50 \mathrm{~cm}$, and a common channel of $280 \mathrm{~cm}$. RYGB was chosen as a less malabsorptive procedure which would allow maintenance of EWL achieved with weight loss surgery.

\section{Discussion}

The true incidence of RFS after bariatric surgery remains unknown, and only three cases of RFS after obesity surgery are described in current literature $[9,10]$. The present case underlines that obese patients with extreme weight loss following obesity surgery associated with undernutrition are at high risk for evolving RFS upon recommencement of increased nutritional intake. Thus, BMI does not play a role as an indicator for patients at risk. 
Chiappettaa and Steinb,c: Refeeding Syndrome: An Important Complication Following Obesity Surgery

It is therefore vital that measures are taken at bariatric centers to identify patients at risk and to effect early diagnosis and prevention. Regular follow-up, constant supplementation, regular monitoring of micronutrient concentrations, and early intervention when weight loss is excessive are of paramount importance. Specific additional risk factors for the development of RFS after obesity surgery include dysphagia and vomiting as well as protracted diarrhea $[9,10]$. Macro- and micronutrient deficiencies are common after obesity surgery [2], and chronic thiamine deficiency can aggravate the risk of evolving RFS.

Metabolic changes due to RFS can cause serious clinical complications since micronutrient and mineral deficiencies impact important physiological functions of the cardiac, respiratory, renal, neurological, hematological, musculoskeletal, and gastrointestinal systems. Possible consequences may include cardiac decompensation, pre-renal failure with metabolic acidosis, and even sudden death [6].

In patients with RFS, use of a feeding jejunostomy in the acute setting is a useful means of promoting protein absorption, especially because it prevents regurgitation of feeding formula. We used a nasogastric tube, placed via upper endoscopy in order to avoid a surgical procedure. While RFS may occur after parenteral or enteral feeding, the risk of RFS is higher after enteral nutrition, probably due to the incretin effect caused by absorption of glucose [11].

The patient described here was in a life-threatening condition, and missed diagnosis would probably have led to the patient's death. Notably, not the BMI itself but the history of weight loss is the major determinant for the identification of patients at risk following obesity surgery. Further research is needed to determine the true incidence of RFS after obesity surgery and to ascertain the best management protocols.

Prevention of RFS through regular follow-up and sufficient supplementation after bariatric surgery is mandatory in all patients.

\section{Conclusion}

Due to chronic weight loss after surgery, obese patients are at high risk for evolving RFS when resuming increased food intake after achieving sufficient weight loss. This applies especially to patients with postoperative complications. RFS is concomitant to any patient suffering from severe malnutrition and requiring feeding, independent of BMI and the bariatric procedure performed. The possibility of RFS as a serious complication must therefore be considered in the long-term postoperative treatment of obese patients.

The identification of patients at risk as well as the prevention and the correct management of this condition should be matters of priority at every bariatric center.

\section{Acknowledgement}

The authors thank Janet Collins (ICCC Rhein-Main, Frankfurt/M., Germany) for language support and proofreading.

\section{Authorship}

Both authors performed substantial contributions to conception and design of the article and to acquisition, analysis and interpretation of data. Both authors reviewed the manuscript for important intellectual content and approved the final version for publication.

Clinical work was performed at Krankenhaus Sachsenhausen, Department of General and Bariatric Surgery (Prof. R. Weiner) and Department of Gastroenterology and Clinical Nutrition, Schulstraße 31, 60594 Frankfurt, Germany. 
Chiappettaa and Steinb,c: Refeeding Syndrome: An Important Complication Following Obesity Surgery

\section{Disclosure Statement}

Dr. Sonja Chiappetta and Prof. Jürgen Stein have no conflicts of interest or financial ties to disclose.

\section{References}

1 Buchwald H, Oien DM: Metabolic/bariatric surgery worldwide 2011. Obes Surg 2013;23:427-436.

2 Stein J, Stier C, Raab H, Weiner R: The nutritional and pharmacological consequences of obesity surgery. Aliment Pharmacol Ther 2014;40:582-609.

3 Scopinaro N, Gianetta E, Adami GF, Friedman D, Traverso E, Marinari GM, Cuneo S, Vitale B, Ballari F, Colombini M, Baschieri G, Bachi V: Biliopancreatic diversion for obesity at eighteen years. Surgery 1996;119:261-268.

4 Santarpia L, Grandone I, Alfonsi L, Sodo M, Contaldo F, Pasanisi F: Long-term medical complications after malabsorptive procedures: effects of a late clinical nutritional intervention. Nutrition 2014;30:1301-1305.

5 Mehanna HM, Moledina J, Travis J: Refeeding syndrome: what it is, and how to prevent and treat it. BMJ 2008; 336:1495-1498.

6 Crook MA: Refeeding syndrome: problems with definition and management. Nutrition. 2014;30: 1448-1455.

7 National Institute for Health and Care Excellence: Nutrition support for adults: oral nutrition support, enteral tube feeding and parenteral nutrition. NICE guidelines CG32. 2006. www.nice.org.uk/guidance/cg32 (last accessed Novemeber 19, 2015).

8 Vignaud M, Constantin JM, Ruivard M, Villemeyre-Plane M, Futier E, Bazin JE, Annane D; AZUREA group (AnorexieRea Study Group): Refeeding syndrome influences outcome of anorexia nervosa patients in intensive care unit: an observational study. Crit Care 2010;14:R172.

9 Baltasar A, del Rio J, Escrivá C, Arlandis F, Martínez R, Serra C: Preliminary results of the duodenal switch. Obes Surg 1997; 7:500-504.

10 Silk Z, Jones L, Heath D: Refeeding syndrome: an important complication after bariatric surgery. Surg Obes Relat Dis 2011;7:e21-23.

11 Zeki S, Culkin A, Gabe SM, Nightingale JM: Refeeding hypophosphataemia is more common in enteral than parenteral feeding in adult in patients. Clin Nutr 2011;30: 365-368. 